\title{
Mosquito antiviral defense mechanisms: a delicate balance between innate immunity and persistent viral infection
}

\author{
Wai-Suet Lee ${ }^{1}$, Julie A. Webster ${ }^{1}$, Eugene T. Madzokere ${ }^{1}$, Eloise B. Stephenson ${ }^{1,2}$ and Lara J. Herrero ${ }^{1 *}$ (D)
}

\begin{abstract}
Mosquito-borne diseases are associated with major global health burdens. Aedes spp. and Culex spp. are primarily responsible for the transmission of the most medically important mosquito-borne viruses, including dengue virus, West Nile virus and Zika virus. Despite the burden of these pathogens on human populations, the interactions between viruses and their mosquito hosts remain enigmatic. Viruses enter the midgut of a mosquito following the mosquito's ingestion of a viremic blood meal. During infection, virus recognition by the mosquito host triggers their antiviral defense mechanism. Of these host defenses, activation of the RNAi pathway is the main antiviral mechanism, leading to the degradation of viral RNA, thereby inhibiting viral replication and promoting viral clearance. However, whilst antiviral host defense mechanisms limit viral replication, the mosquito immune system is unable to effectively clear the virus. As such, these viruses can establish persistent infection with little or no fitness cost to the mosquito vector, ensuring life-long transmission to humans. Understanding of the mosquito innate immune response enables the discovery of novel antivectorial strategies to block human transmission. This review provides an updated and concise summary of recent studies on mosquito antiviral immune responses, which is a key determinant for successful virus transmission. In addition, we will also discuss the factors that may contribute to persistent infection in mosquito hosts. Finally, we will discuss current mosquito transmission-blocking strategies that utilize genetically modified mosquitoes and Wolbachia-infected mosquitoes for resistance to pathogens.
\end{abstract}

Keywords:: Mosquito, Antiviral defense, RNAi pathway, Persistent infection, Transmission-blocking strategies

\section{Background}

Mosquito-borne viruses are a global health priority due to frequent resurgences of major epidemics and unprecedented geographical expansion in the last few decades [1]. The majority of mosquito-borne viruses are RNA viruses, with either single or double-stranded RNA that have positive or negative polarity. Viruses primarily associated with global morbidity and mortality are from the following families: Flaviviridae (genus Flavivirus, positive single-stranded RNA), Togaviridae (genus Alphavirus, positive single-stranded RNA) and Bunyaviridae (genus Phlebovirus, negative single-stranded RNA) [2,

\footnotetext{
*Correspondence: I.herrero@griffith.edu.au

${ }^{1}$ Institute for Glycomics, Griffith University, Gold Coast Campus, Southport, QLD 4215, Australia

Full list of author information is available at the end of the article
}

3]. Viruses from the Flaviviridae family include yellow fever virus (YFV), dengue virus (DENV), Japanese encephalitis virus (JEV), West Nile virus (WNV) and Zika virus (ZIKV) [4]. DENV is considered the most important mosquito-borne virus, causing 390 million dengue infections annually, and is primarily transmitted by Aedes aegypti [5]. Dengue epidemics have expanded significantly in the last few decades to at least 128 countries [6]. Aedes aegypti is also the primary vector for other flaviviruses, including YFV and ZIKV. Zika virus infections are typically mild or asymptomatic, but have been linked to Guillain-Barré syndromes in adults. Zika virus infections are also a major concern to pregnant women and are associated with birth defects, such as microcephaly in prenatally infected infants [7]. In February 2016, ZIKV epidemics were declared a public health 
emergency by the World Health Organization (WHO), with 62 countries and territories reporting evidence of transmission since its introduction into Brazil in 2015 [8, 9]. Some flaviviruses, including JEV and WNV, are primarily transmitted by mosquitoes belonging to the genus Culex $[8,10]$. Members that belong to the Togaviridae family include chikungunya virus (CHIKV), Sindbis virus (SINV), Semliki Forest virus (SFV) and Ross River virus (RRV) [11]. The Anopheles mosquito, which is the main vector for the parasite Plasmodium falciparum, is only known to transmit O'nyong-nyong virus (ONNV), which belongs to genus Alphavirus [12].

Following ingestion of a viremic blood meal from an infected vertebrate host, the virus initiates infection in the mosquito midgut. However, the dissemination of virus from the midgut to salivary glands is not well understood. It is postulated that once the virus enters midgut epithelial cells, replication occurs in the cells and the virus subsequently spreads to the hemocoel [13]. The hemocoel is an open body cavity where hemolymph circulates, and thus once the hemolymph is inoculated, the virus can spread to other secondary tissues via hemolymph circulation, including the salivary gland, fat body, trachea, muscles and neural tissue $[14,15]$. The spread of virus to the salivary glands is essential for the mosquito to be competent for virus transmission to subsequent vertebrate hosts $[16,17]$.

\section{The mosquito innate immune pathways in mosquitoes}

The mosquito innate immune response is a key determinant for successful transmission of viruses. Unlike the mammalian immune system, mosquitoes do not possess adaptive immune responses and are dependent on innate immunity to fight viral infection. Most of the knowledge on insect antiviral innate immunity is elucidated from studies of the genetic model insect Drosophila melanogaster $[18,19]$. Viral infection triggers the activation of innate immunity pathways and leads to the transcription of genes responsible for antiviral responses.

The innate immune system of mosquitoes consists of two tightly interconnected responses: the cellular and humoral defense responses. These two responses act together to protect mosquitoes against a wide variety of pathogens, including bacteria, yeast and viruses. The cellular defense response includes phagocytosis, nodulation and encapsulation of pathogens by hemocytes [20, 21]. Humoral responses refer to the activation of downstream signaling and effector responses, leading to the synthesis and secretion of soluble effector molecules, such as antimicrobial peptides (AMPs), reactive oxygen species (ROS) and components of the phenoloxidase cascade [14, 22-25]. Downstream signaling and humoral effector responses will be discussed later in this review. These effector molecules are secreted into the hemolymph to control infection caused by invading pathogens $[26,27]$. Epithelial cells in the mosquito midgut are the first line of defense against many pathogens which are acquired from blood-feeding and these cells can synthesize several AMPs and ROS. Additionally, the fat body of the mosquito is the primary site of the humoral response via production and secretion of AMPs. The transcription of innate immunity genes encoding for AMPs is highly dependent on several signaling cascade pathways, including the Janus kinase-signal transducer and activator of transcription (JAK-STAT), Toll and immune deficiency (Imd) pathways [9, 14, 25, 28, 29]. Although activation of these pathways has been shown to limit viral replication, the most robust antiviral defense is the RNA interference (RNAi) pathway. The RNAi pathway produces small RNAs using viral double-stranded RNA as a template to ultimately target the viral RNA for degradation, thereby inhibiting viral replication [30]. Virus recognition is mediated by pattern recognition receptors (PRRs) that recognize virus-conserved pathogen-associated molecular patterns (PAMPs) to initiate innate immune responses.

\section{RNA interference (RNAi) pathways}

RNA interference (RNAi) is the central antiviral mechanism in insects, particularly in controlling virus infection through degradation of RNA, also known as RNA silencing. The key event in the RNAi pathway is the production of small RNAs from long viral double-stranded RNA (dsRNA) (Fig. 1) [31]. There are three major types of small RNAs: (i) small interfering RNAs (siRNAs), (ii) microRNAs (miRNAs), and (iii) PIWI-interacting RNAs (piRNAs), with siRNAs being the main antiviral responses in mosquito. Mosquito-borne viruses are primarily RNA viruses, with their genomes comprised of single-stranded RNA that is either positive-sense $(+)$ or negative-sense (-) [30,32]. During genome replication, these viruses generate dsRNA as replication intermediates [33]. The siRNA pathway is responsible for the major antiviral response [34]. The viral dsRNA binds to a Dicer-2-R2D2 complex, which consists of a RNase III enzyme, called Dicer-2 (Dcr-2), and an associated protein, called R2D2 [35]. The dsRNA is cleaved by the RNase III domain of Dcr-2 to produce siRNAs of 21-23 nucleotides (nt) in length [36]. The siRNAs then activate the RNAi machinery by binding to another multiprotein known as the RNA-induced silencing complex (RISC), in which one of the RNA strands is degraded. The remaining single-stranded RNA (ssRNA) then serves as a guidestrand to detect and degrade cognate viral RNA by host endonuclease, Argonaute-2 (Ago2) in a sequence specific 


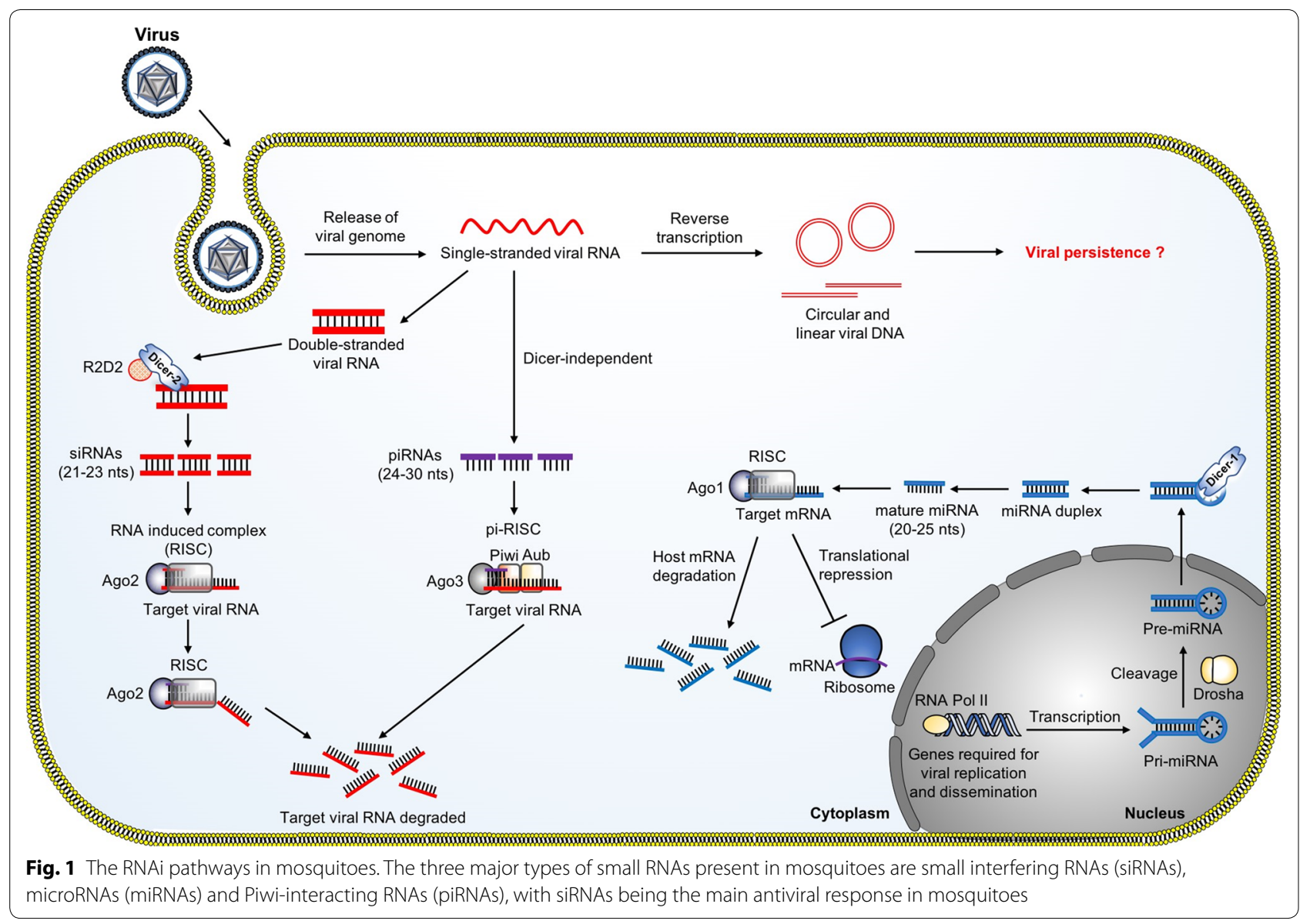

manner [31]. One study has demonstrated that silencing of the RNAi pathway during DENV2 infection in $A e$. aegypti enhanced virus replication, indicating their role in controlling viral replication [37]. Transgenic Ae. aegypti mosquitoes with RNAi pathway impairment in the midgut were observed to have enhanced SINV replication in the midgut and increased virus dissemination rates [38]. In DENV-infected Ae. aegypti, virus-specific siRNAs (20-23 nt), piRNAs (24-30 nt) and unusually small RNAs (13-19 nt) were detected [39]. The siRNA pathway is also an elicited antiviral response in An. gambiae against ONNV infection [40].

miRNAs are a class of endogenous small non-coding RNAs (20-25 nt) and play significant roles in the posttranscriptional regulation of target genes in multiple metabolic processes by cleavage of target mRNAs or repression of mRNA translation [41, 42]. Similar to the siRNA pathway, the miRNA pathway starts with cleavage of the dsRNA into small dsRNA, which is loaded into the RISC and serves as a guide-strand to detect and degrade cognate viral ssRNA. The differences between the siRNA and miRNA pathways are the cellular compartments and the effector proteins involved in the pathways [43]. The transcription, cleavage and processing of siRNA mainly take place in the cytoplasm while the miRNA genes are transcribed into primary miRNA (pri-miRNA) by host polymerase II and are processed into precursor miRNA (pre-miRNA) by Drosha in the nucleus. The pre-miRNA is then exported into the cytoplasm and further processed into a mature miRNA by Dicer-1 and is loaded into Ago- 1 of the RISC, which guides the binding of the complex to complementary mRNA for degradation [44]. The antiviral role of miRNAs in mosquitoes has not been reported as it was assumed that RNA viruses do not generate miRNAs. This is because of a lack of access to the Drosha for miRNA processing in the nucleus as replication of most RNA viruses occurs in the cytoplasm [45, 46]. However, miRNAs from a number of arbovirus mosquito vectors have been shown to play a critical role in modulating host genes to control viral infection. For example, several miRNAs specific for innate immunity and multiple metabolic processes required for viral replication and dissemination were modulated during ZIKV [47], DENV [48] WNV [49] and ONNV infections [50].

Besides the well-studied siRNA pathway, recent studies have highlighted the importance of the piRNA pathway 
in the mosquito antiviral response [51-53]. Interestingly, the piRNA pathway can mount an antiviral defense with a defective siRNA pathway, indicating the redundancy of RNAi-mediated antiviral immune responses [51]. In contrast to siRNAs, the biogenesis of piRNAs does not require Dicer and the size distribution of piRNAs is around 24-30 nt [54]. In Drosophila, the biogenesis of piRNAs involves three Piwi proteins, including the P-element induced wimpy testis (Piwi), Aubergine (Aub) and Argonaute 3 (Ago3), to form a piRNA-induced silencing complex (piRISC) [55]. The biogenesis starts with the primary processing pathway, which is the synthesis of primary piRNA pool from single-stranded precursors. The primary piRNAs can be associated with the Aub and Piwi protein. Interestingly, the primary pool of piRNAs can undergo an amplifying process known as the 'pingpong' cycle to further amplify the Aub-bound piRNAs to refine the piRNA pool. This amplification process serves to ensure an efficient piRNA-mediated silencing of target RNA [52-54]. The presence of virus-specific piRNAs was detected in Ae. aegypti and Ae. albopictus during CHIKV infection [56] and in SINV infected Aedes cells [53]. Deep sequencing data reported the presence of SFV-derived piRNAs and silencing of PIWI 4 protein resulted in increased SFV replication and virion production, suggesting the importance of the piRNA pathway in antiviral immunity [57].

\section{Viral DNA produced during replication is important for mosquito survival and persistent infection}

The mosquito immune response is implicated in virus persistence [30, 58, 59]. Despite activation of mosquito antiviral immune responses during viral infections, viruses are not completely eliminated from the mosquitoes. Instead, a persistent infection, with little or no cost of fitness to the host, is established in mosquitoes, which makes them efficient vectors for viral diseases. However, the mechanisms by which viruses maintain persistent infection in mosquitoes are poorly understood. Recent studies have demonstrated that virusderived DNA (vDNA) generated during viral infection are important for mosquito survival and persistent infection $[30,58]$. The majority of mosquito-borne viruses are RNA viruses, and upon infection, viral RNA or truncated forms of the viral genome produced during virus replication, also known as the defective viral genomes (DVGs), are reverse transcribed to vDNA by the activity of host cellular reverse transcriptase [58]. Although the biogenesis and regulation of vDNAs in mosquitoes has not been well studied, it has been reported that Dcr- 2 regulates the production of vDNA from DVGs as illustrated in Fig. 1. Dcr-2 is a multifunctional protein and vDNA production is regulated by its $\mathrm{DExD} / \mathrm{H}$ helicase domain [58]. vDNAs can be detected not only in mosquito cell culture during infection, but also in Ae. aegypti and Ae. albopictus during CHIKV and ZIKV infections [30, 59]. The vDNAs then stimulate the RNAi machinery to control viral replication. Interestingly, vDNA is sufficient to produce siRNAs to elicit antiviral response when challenged with a cognate virus. Furthermore, inhibition of vDNA production results in extreme susceptibility to viral infections [30].

\section{Other innate immune pathways: JAK-STAT, Toll and Imd pathways}

In addition to the RNAi pathway, there are other innate immune pathways involved in protecting mosquitoes against viral infection, including the JAKSTAT, Toll and Imd pathways (Fig. 2). In response to viral infection, activation of these pathways initiates the formation of a multiprotein complex consisting of protein kinases, transcription factors and other regulatory molecules to regulate the expression of downstream innate immunity genes [14, 22, 60]. These include genes that encode for AMPs and key factors that regulate the innate immune response to viruses. AMPs are immune-inducible peptides that are potent and rapid-acting immune effectors with antimicrobial activities [61]. A wide spectrum of AMPs have been reported in insects during infection with Gram-negative and Gram-positive bacteria, filamentous fungi and yeast $[19,61]$. These AMPs carry out both direct killing and innate immune modulation (recruitment and activation of immune cells) to limit invading pathogens $[19,62,63]$. Most studies on the regulation of AMPs during infection are based on Drosophila, and the regulation of AMPs in mosquitoes is poorly understood. AMPs vary among different mosquito species and the induction of AMPs is regulated by multiple immune signaling pathways and is highly dependent on the type of pathogen that elicited the response. In Ae. aegypti, 17 AMPs have been identified, and they belong to five different families: defensins (cysteinerich peptides), cecropins ( $\alpha$-helical peptides), diptericin (glycine-rich peptides), attacin (glycine-rich peptides) and gambicin (cysteine-rich peptides) [61, 63, 64]. The mode of killing of AMPs is often specific for different microorganisms. Defensins are active and highly toxic against Gram-positive bacteria and parasites by disrupting the membrane permeability barrier, thereby causing loss of motility [65]. As for cecropins, these positively charged peptides bind to the lipids in the membrane that are negatively charged, thus changing the biological structure of membranes. Other possible modes of killing by cecropins include inhibition of nucleic acid and protein synthesis and inhibition 


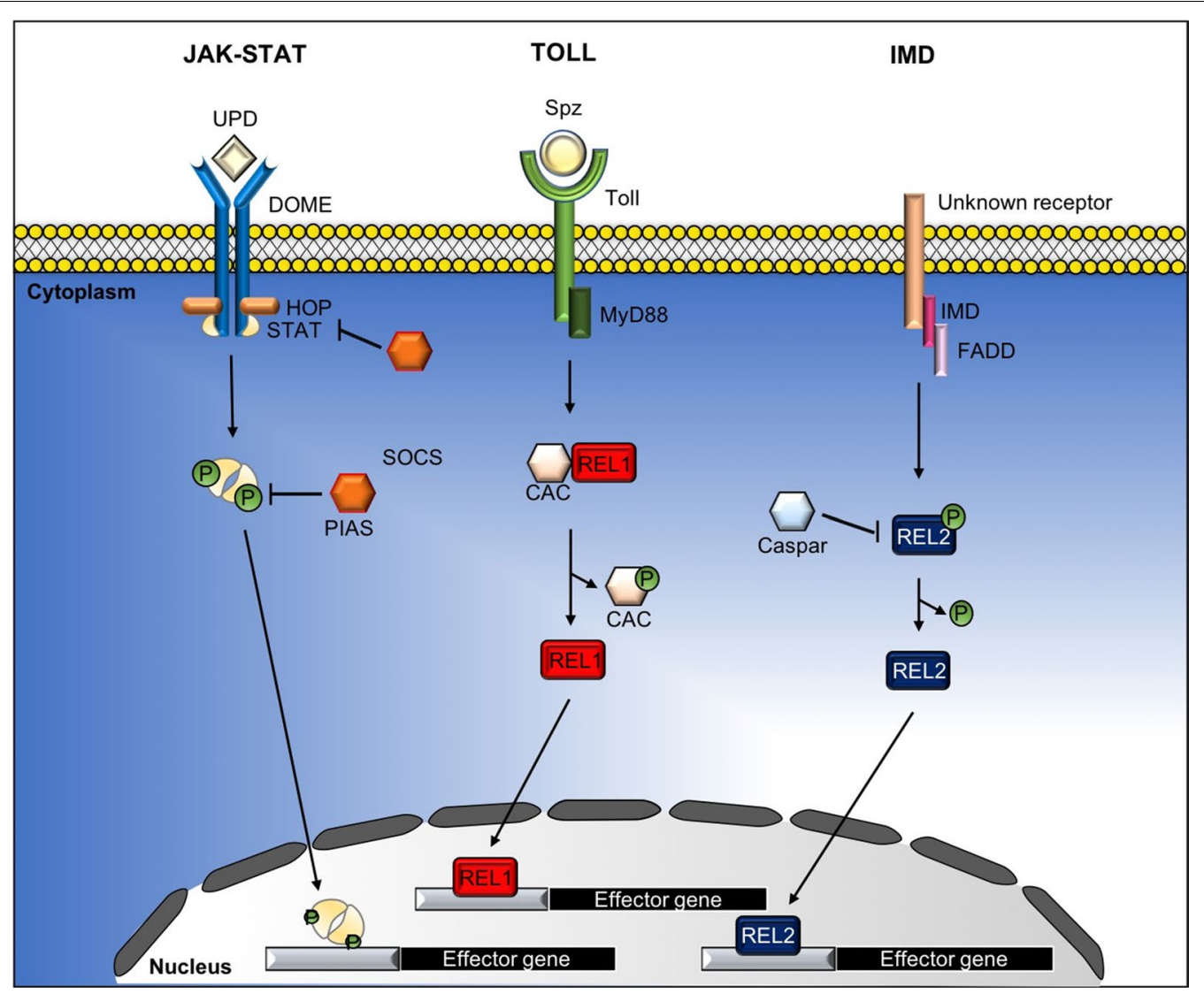

Fig. 2 The JAK-STAT, Toll and immune deficiency (Imd) pathways in mosquitoes. Activation of the JAK-STAT, Toll and Imd pathways initiates the formation of a multiprotein complexes consisting of protein kinases, transcription factors and other regulatory molecules to regulate the expression of downstream innate immunity genes, such as the genes that encode for AMPs and key factors that regulate the innate immune system

of enzymatic activity [66]. Defensins and cecropins have been found to be expressed in the midgut, thorax and abdominal tissues of An. gambiae mosquitoes and are induced during infection with parasite [67]. In the same mosquito species, gambicin has been found to be induced by parasites in the midgut, fat body and hemocytes [64]. However, their role in regulating antiviral immune response is not completely understood. In Ae. aegypti, cecropins are upregulated in DENV-2 infected mosquitoes [66]. Furthermore, cecropins exhibit antiviral activity against DENV and CHIKV [66]. In Culex mosquitoes, Vago is a secreted peptide regulated by the JAK-STAT pathway and overexpression of Vago reduces the viral load of WNV in mosquitoes [28]. During SINV infection in Drosophila, two AMPs, regulated by the Imd and the JAK-STAT pathways, namely the attacin $C$ and diptericin $B$, control viral RNA synthesis and knocking down of these genes increases viral load in flies [10].

\section{The JAK-STAT pathway}

The JAK-STAT pathway was originally identified in Drosophila and was shown to have an active role in antiviral defense against Drosophila $\mathrm{C}$ virus (DCV) and Flock House virus (FHV) [18]. Consistent with Drosophila, mosquitoes also express the cytokine receptor, Domeless (Dome) and the tyrosine kinase Hopscotch (Hop), which together induce the JAK-STAT pathway. The mechanism of the Dome receptor is similar to the mammalian JAK-STAT pathway. The ligand binds to Dome, which then undergoes a conformational shift leading to self-phosphorylation of Hop (JAK). Activated Hop phosphorylates Dome, which forms a docking site for cytosolic STATs. Recruitment of STATs by the Dome/Hop complex leads to the phosphorylation and release of the STATs. The phosphorylated STATs dimerize and translocate to the nucleus where they activate transcription of specific effector genes, such as the virus-induced RNA 1 (vir-1) gene, that has a role in antiviral immunity [18, 22]. Through reverse genetic approaches and functional 
studies, the JAK-STAT pathway has been shown to mediate increased resistance to DENV and ZIKV in infected Ae. aegypti [22, 25]. Genetically modifying Ae. aegypti to overexpress Dome and Hop renders the mosquitoes more resistant to DENV infection, but not to CHIKV and ZIKV infections. These studies suggest that Ae. aegypti possess varied molecular responses to different viruses [68].

The Dome receptor is the most well characterized cytokine receptor in mosquitoes; however, evidence suggests that other cytokine receptors are present which also activate the JAK-STAT pathway. For example, in Culex mosquitoes, a secreted peptide known as Vago was upregulated in response to WNV infection, subsequently activating the JAK-STAT pathway to control infection and reduce viral load [28]. However, knockdown of Dome did not inhibit signaling of the JAK-STAT pathway, indicating that Vago activated JAK-STAT via another unknown receptor [28].

Transgenic Ae. aegypti mosquitoes have been used to investigate the role of the JAK-STAT pathway in viral infection. Through RNAi-mediated gene silencing of the tyrosine kinase complex, Dome and Hop increased DENV infection, whereas knockdown of PIAS, a known negative regulator of the JAK-STAT pathway, decreased DENV infection [22]. However, although the JAK-STAT pathway is increased in response to DENV infection in the mosquito, strains that were either resistant or susceptible to DENV infection did not show a difference in viral infection, indicating that the pathway was not involved in viral susceptibility to DENV [69].

The majority of investigations into the JAK-STAT pathway in mosquito immunity have involved dengue infection; however, pathway activation in response to other viruses and downstream mechanisms may differ for each virus. Transgenic overexpression of Hop in the midgut decreased DENV2 infection and dissemination; however, for ZIKV, dissemination was only decreased at day 7 post-infection and infection was not altered [68]. In contrast to ZIKV and DENV, the JAK-STAT pathway was not activated by CHIKV infection [70], nor was it involved in viral dissemination [68]. Furthermore, in human host cells, CHIKV non-structural protein 2 has been shown to inhibit interferon signaling via inactivation of the JAK-STAT pathway [71]; however, the precise mechanism of action has not been elucidated. Together, this raises the possibility that the CHIKV inhibitory mechanism acts directly on the JAK-STAT pathway and hence may be conserved in the mosquito immune system. Just as CHIKV may inhibit the JAK-STAT pathway, SFV has also been shown to downregulate transcription of the JAK-STAT pathway [9]. Thus, both CHIKV and SFV have developed mechanisms to avoid activation of this pathway and the downstream effectors of the JAK-STAT pathway are differentially affected between the viruses.

\section{The NF-kB-like signaling pathways: Toll and Imd pathways}

The Toll and Imd pathways are two distinct innate immune pathways very similar to the mammalian $\mathrm{NF}-\mathrm{KB}$ signaling pathway, which is the key regulator in the production of AMPs. The Toll pathway was first reported in Drosophila, and is known for its role in innate immunity against pathogens, such as fungi and Gram-positive bacteria [72]. In contrast, the Imd pathway is activated during infection by Gram-negative bacteria [72]. Both Toll and Imd pathways are activated by pathogens via binding of PAMPs to the host's PRRs, which leads to a cascade of events to activate immune effector genes for production of AMPs. The Toll pathway is initiated by cleavage of the cytokine Spätzle (Spz), which is a ligand that binds to the Toll transmembrane receptor. Activated Toll triggers signaling through MyD88, Tube (adaptor proteins associated with Toll) and the Pelle kinase. Subsequently, the negative regulator of the Toll pathway, Cactus, is phosphorylated and undergoes proteasomal degradation that cause the translocation of the transcription factor Relish 1 (Rel1) from the cytoplasm to the nucleus and binding to $\mathrm{kB}$ motifs on the promoters of many AMPs genes, such as Diptericin and Cecropin that are active against fungi and Gram-positive bacteria [73]. While in the Imd pathway, activation of the pathway leads to degradation of the negative regulator Caspar, which leads to the translocation of Relish 2 (Rel2) to the nucleus, resulting in the transcription of AMPs [14, 74].

The majority of studies on the Toll and the Imd pathways are focused mainly on their antifungal and antibacterial functions in mosquitoes [73]; however, their role in antiviral immune response is not well characterized. Comparative genomic analysis between Drosophila and mosquitoes revealed that the key components of the Toll and the Imd pathways are conserved between these two species. The homologues of genes from the Toll and the Imd pathways can be found in Ae. aegypti, Cx. quinquefasciatus and An. gambiae. During DENV infection of Ae. aegypti, the genes in the Toll pathway (GNBP, Toll5A and MYD88 genes) were upregulated in the salivary glands. Silencing of MYD88, caused a slight increase of DENV viral titre in the midgut [66]. Upon viral infection, Rel1 and its downstream antimicrobial peptides is upregulated to control infection against DENV $[14,75]$ and SINV [24], whereas in Culex mosquitoes, following WNV infection, the transcription factor Rel2 of the Imd pathway activates the secretion of an antiviral peptide against WNV infection [28]. 


\section{The Delta-Notch signaling pathway: a complementary pathway in regulating antiviral immunity}

The evolutionarily conserved signaling pathway, DeltaNotch, plays crucial roles in embryonic development, stem cell maintenance and adult tissue renewal [76]. While the Delta-Notch signaling pathway was well described for its role in developmental processes, a recent study has reported a new role of the Delta-Notch signaling pathway in antiviral innate immunity in the mosquito, by limiting the replication of DENV in Ae. aegypti mosquitoes [77]. Notch is a transmembrane receptor and signaling depends on the binding of Delta ligands, which activates the proteolysis of the Notch receptor, releasing an active fragment, known as the Notch intracellular domain (NICD) that enters the nucleus to activate downstream target genes [76, 78]. During DENV infection, components of this pathway including Delta, Notch and Hindsight genes were also shown to be upregulated in $A e$. aegypti mosquitoes [77]. Although the exact mechanism of how this signaling pathway limits DENV replication is not known, this study showed that activation of this signaling pathway induced endoreplication, in which cells undergo many rounds of DNA replication without mitosis to increase dramatically the genomic DNA content in the cells. Induction of endoreplication increased the number of gene transcripts that are involved in controlling viral spread [77].

\section{The cellular immune mechanisms in mosquitoes}

The cellular defense response includes phagocytosis, nodulation and encapsulation of pathogens by hemocytes [20]. Furthermore, hemocytes also elicit humoral responses by activation of downstream signaling as previously mentioned and their effector responses lead to the synthesis and secretion of soluble effectors molecules such as AMPs and components of the phenoloxidase cascade into the hemolymph to control infection against invading pathogens [79].

\section{Hemocyte-mediated antiviral immunity in mosquitoes}

Hemocytes are cells that circulate within hemolymph, and are permissive to viral infection including DENV [15], SINV [80] and WNV [81]. The hemocyte-mediated immune response is immediate and includes pattern recognition, phagocytosis, nodulation, melanization, production of antimicrobial peptides and initiation of signaling cascades for cytotoxic effectors to clear infection $[20,80,82]$.

Hemocytes exist in two forms: circulating (circulate within hemolymph) and sessile (tissue resident). Furthermore, different populations of hemocytes have been described in mosquitoes. Studies have categorized mosquito hemocytes into prohemocytes, oenocytoids and granulocytes [83]. Granulocytes are the most abundant and constitute $80-95 \%$ of circulating hemocytes. Granulocytes are phagocytic, and upon activation they rapidly adhere to and engulf foreign particles [84]. Oenocytoids ( $10 \%$ of circulating hemocytes) are the main producer of components of the phenoloxidase (PO) cascade in response to infection [85]. The PO cascade is a humoral immune response initiated by pathogen-associated pattern recognition molecules and leads to proteolytic processing of prophenoloxidase (PPO) to $\mathrm{PO}$, which catalyzes the formation of melanin around invading pathogens [86]. The reaction intermediates generated from the proteolytic processes have been shown to inactivate SFV [86]. SFV has been shown to activate PO-based melanization cascade in mosquito cells, which results in inhibition of virus spread indicating that this pathway mediates the antiviral response in mosquitoes [86]. Nodulation occurs when multiple hemocytes bind to bacterial aggregates to form a multicellular sheath and the nodule formation is the main insect cellular defense reaction to clear a large number of bacteria from the hemolymph [20,21].

\section{Fat body-mediated antiviral response of mosquitoes}

The insect fat body is an organ that functions analogous to both adipocytes and livers in mammals. The fat body is crucial in regulating metabolism and growth in insects, and is responsible for energy storage, synthesis and secretion of hemolymph proteins and circulating metabolites [87]. A recent study reported that the JAK-STAT pathway is activated in the fat body of Ae. aegypti during dengue virus infection [68]. Overexpression of the Dome or Hop gene in the fat body of Ae. aegypti, resulted in inhibition of DENV infection in these transgenic mosquitoes, but this inhibitory effect was not observed for CHIKV and ZIKV, indicating that different viruses elicited the JAKSTAT pathway differently [68].

As the fat body is important in mediating antiviral responses in mosquitoes, its components such as cellular lipids may play a role as well. It has been shown that cellular lipids are manipulated by flaviviruses to facilitate viral replication. In Ae. aegypti cells, SINV and DENV infection resulted in accumulation of lipid droplets (LDs) [88]. LDs are made up of a monolayer of fatty acid and other structural proteins including Perilipin 1, 2 and 3. LDs are found in the fat body tissue of mosquitoes and their main function is maintaining lipid homeostasis, by regulating biogenesis and degradation of LDs [88]. LDs serve as a reservoir of lipids which are important for anchoring the viral replication machinery for efficient viral replication [89]. Exploitation of lipid metabolism has also been reported in WNV, indicating the importance of lipids in pathogenesis [90]. Genes involved in LD biogenesis and lipid metabolism 
are upregulated upon DENV infection [88]. Interestingly, activation of immune signaling pathway, including the Toll and the Imd pathways enhanced LD content in mosquito midgut [88]. During DENV infection, fatty acid synthase is recruited to the site of replication by DENV nonstructural protein 3 to stimulate fatty acids synthesis [91]. Furthermore, inhibition of fatty acid synthase decreased DENV viral titers and thus serve as a potential antiviral target to control viral infections [92].

\section{Autophagy to promote antiviral immunity}

Autophagy is an evolutionarily conserved process that sequesters and mediates the degradation of cellular components, such as proteins and organelles, to maintain cellular and tissue homeostasis [93]. Autophagy involves the sequestration of damaged organelles or misfolded proteins by forming double-phospholipid membrane vesicles, known as autophagosomes. The autophagosomes then fuse with lysosomes to mediate the degradation of sequestered contents within the lysosome $[93,94]$. In addition to the role of autophagy in maintaining cellular and tissue homeostasis, a protective role for autophagy against intracellular pathogens including viruses has been shown in mammalian systems and, to a lesser extent, in Drosophila [95-97]. In Drosophila, antiviral autophagy against vesicular stomatitis virus (VSV) and Rift Valley fever virus (RVFV) is activated through pathogen recognition by the Toll-7 transmembrane receptor. The activation of Toll-7 leads to the activation of autophagy via the phosphatidylinositol 3-kinase (PI3K)-Akt-signaling pathway, which is an autophagy pathway that senses the status of nutrient availability. Upon activation, autophagy is able to limit viral replication in flies. Furthermore, loss of Toll-7 leads to an increase in viral RNA production in Drosophila cell line [97] and Toll-7 mutant flies which are more susceptible to RVFV infection [95, 96], suggesting that there is a role for autophagy in controlling viral replication. Due to the conservation of autophagy, it is postulated that the autophagy pathway is also involved during viral infection of mosquitoes. For example, during DENV infection, autophagy is activated to generate energy for viral replication. In particular, autophagy regulates lipid metabolism by degradation of the lipid droplets to release lipids that undergo oxidation to generate energy for viral replication [91, 98]. However, the role of autophagy during virus infection of mosquitoes is still largely unknown.

\section{Current mosquito control strategies}

The prevention and control of mosquito-borne diseases is primarily reliant on vector control measures, such as the use of insecticides, mosquito nets and environmental management to limit human-vector contact [5]. Over the last decade, approaches such as the release of Wolbachiainfected mosquitoes $[99,100]$ and genetically modified mosquitoes [101, 102] into native mosquito populations have been undertaken. These approaches aim to either reduce viral capacity in vector populations or reduce reproductive success.

\section{Wolbachia-infected mosquitoes}

Wolbachia pipientis are symbiotic bacteria, vertically transmitted from mother to offspring, and exist naturally in an estimated $60 \%$ of insects [103]. Recently, the $w$ Mel strain of Wolbachia has been introduced into Ae. aegypti, which is not a natural host of Wolbachia, in an attempt to limit their ability to transmit important arboviruses including DENV, CHIKV and ZIKV. To date, ten countries, including Australia, Brazil and Vietnam, have participated in field trials for DENV control by releasing Wolbachia-infected mosquitoes into the wild [104]. In controlled field releases in Cairns, Australia, the $w \mathrm{Mel}$ strain of Wolbachia was successfully established in natural populations of Ae. aegypti mosquitoes [100]. Several years later, the Wolbachia infection rate in the mosquito population remains high [105]. Additionally, Wolbachiainfected mosquitoes from the same field populations continue to demonstrate reduced susceptibility to DENV under laboratory conditions [106]. Field and clinical studies in Vietnam showed that wMel-infected Ae. aegypti are not permissive to DENV infection when the mosquitoes were fed with patient-derived viremic blood meals [107].

Despite the potential of Wolbachia as a useful and effective tool to combat mosquito-borne diseases, the mechanisms of how Wolbachia mediate viral replication in mosquitoes remains largely unclear. However, there are likely to be multiple mechanisms involved: (i) priming the immune system by inducing reactive oxygen species (ROS) and activating innate immune genes to secrete effector proteins such as Vago to limit viral replication [108, 109]; (ii) direct competition for cholesterol between viruses and Wolbachia [110]; and (iii) perturbations in vesicular trafficking, lipid metabolism, intracellular cholesterol trafficking and in the endoplasmic reticulum (ER) [111]. Despite promising results from field trials, many concerns need to be addressed before Wolbachia-infected mosquitoes can become a safe and effective strategy to suppress arbovirus transmission. For example, one study has reported that Wolbachia-infection of Ae. aegypti increased the infection rates of other insect-specific flaviviruses that are not medically important [112]. Secondly, Wolbachiabased mosquito control might not be effective for other mosquito species. For example, Wolbachia-infection of $C x$. tarsalis, which is a novel WNV vector in North 
America, enhanced the infection rate of WNV [113]. Additionally, Wolbachia-infected Anopheles mosquitoes exhibited an enhanced susceptibility to Plasmodium infection, thus increased the risk of malaria transmission by these mosquitoes [114].

\section{CRISPR/Cas9 genetically-modified mosquitoes}

The clustered regularly interspaced short palindromic repeats/CRISPR associated sequence 9 (CRISPR/Cas9) system has recently emerged as a powerful genome editing tool to combat vector-borne diseases [115, 116]. In addition, the use of the CRISPR/Cas9 system to genetically modify mosquitoes to combat mosquitoborne diseases is steadily growing. For example, the body of literature on the development of highly effective CRISPR/Cas9 systems has expanded significantly especially within the last five years since the emergence of this system. Briefly, the CRISPR/Cas9 system comprises two components: (i) a small RNA (17-20 bases) known as single guide RNA (sgRNA) that is designed to complement the target genomic DNA sequence; and (ii) Streptococcus pyogenes Cas9 nuclease (SpCas9) that binds and cleave the double-stranded target DNA in the presence of a short conserved sequence (2-6 nucleotides), known as protospacer-associated motif (PAM) [115]. The SpCas9 endonuclease complexes with the sgRNA and induces double-stranded DNA breaks at the target DNA sequence [115]. The PAM sequence for SpCas9 is NGG, and SpCas9 will not bind to the target DNA sequence if PAM is absent at the site. Interestingly, the frequency of NGG in the Ae. aegypti genome is relatively high (approximately once every 17 base pairs). This feature makes CRISPR/CAS9 an efficient and reliable system to make precise changes to the genome of this vector species $[115,117]$.

Recently, CRISPR/Cas9-based gene editing has been widely used as an efficient tool to modify the mosquito genome of An. stephensi [116], Ae. aegypti [115], Cx. quinquefasciatus [118] and Cx. pipiens [119]. More recently, CRISPR/Cas9 has been used to generate a knockout mutant of the fibrinogen-related protein 1 (FREP1) gene of An. gambiae, which encodes for a specific immune protein that is important for parasite's midgut infection stage to block malaria transmission [101].

\section{Conclusions}

Currently there are no suitable vaccines nor cure for the majority of mosquito-transmitted diseases. Vector control remains the gold standard strategy to block disease transmission. More recently, genetically-modified mosquitoes have been developed and field tests are ongoing, as potential alternative strategies to control disease transmission by mosquitoes. However, these strategies are not perfect and insufficient to block transmission. Furthermore, as these strategies are still novel, little is known about how viruses and mosquito defense mechanisms may evolve to reduce the efficacy of these strategies. More extensive knowledge of how mosquitoes respond to infection, how the innate immune system controls virus infection, other host factors that facilitate viral replication, how viruses persist in mosquitoes and how different mosquito species or strains vary in permissiveness to virus infection at the molecular level could improve and maximize the effectiveness of current strategies and could possibly result in identification of new molecular targets for new vector control strategies.

\section{Abbreviations}

PRRs: pattern recognition receptors; PAMPs: pathogen-associated molecular patterns; YFV: yellow fever virus; DENV: dengue virus; JEV: Japanese encephalitis virus; WNV: West Nile virus; ZIKV: Zika virus; CHIKV: chikungunya virus; SINV: Sindbis virus; SFV: Semliki Forest virus; RRV: Ross River virus; ONNV: O'nyongnyong virus; AMPs: antimicrobial peptides; ROS: reactive oxygen species; JAK-STAT: Janus kinase-signal transducer and activator of transcription; Imd: immune deficiency; RNAi: RNA interference; siRNAs: small interfering RNAs; miRNAs: microRNAs; pri-miRNA: primary microRNA; pre-miRNA: precursor microRNA; piRNA: Piwi-interacting RNA; dsRNA: double-stranded RNA; Dcr-2: Dicer-2; RISC: RNA-induced silencing complex; ssRNA: single-stranded RNA; Ago2: Argonaute-2; Piwi: P-element induced wimpy testis; Aub: Aubergine; Ago3: Argonaute 3; piRISC: piRNA-induced silencing complex; VSV: vesicular stomatitis virus; CRISPR/Cas9: clustered regularly interspaced short palindromic repeats/CRISPR associated sequence 9; sgRNA: single guide RNA.

\section{Acknowledgements}

We thank Dr Bernadette Bellette for providing a critical review of the manuscript. LHH is the recipient of the Australian National Health and Medical Research Council Career Development Award (ID: 105760).

\section{Funding}

This work was supported by the Australian National Health and Medical Research Council grant and ARC Discovery Early Career Researcher Award to LJH (Grant ID: 1081954 and DE140101493).

Availability of data and materials Not applicable.

\section{Authors' contributions}

WSL and LJH conceived, designed, edited and performed final revisions on the manuscript. WSL, JAW, ETM and EBS wrote and edited the manuscript. All authors read and approved the final manuscript.

Ethics approval and consent to participate Not applicable.

\section{Consent for publication} Not applicable.

\section{Competing interests}

The authors declare that they have no competing interests.

\section{Publisher's Note}

Springer Nature remains neutral with regard to jurisdictional claims in published maps and institutional affiliations. 


\section{Author details}

${ }^{1}$ Institute for Glycomics, Griffith University, Gold Coast Campus, Southport, QLD 4215, Australia. ${ }^{2}$ Environmental Futures Research Institute, Griffith University, Gold Coast Campus, Southport, QLD 4215, Australia.

Received: 13 November 2018 Accepted: 4 April 2019 Published online: 11 April 2019

\section{References}

1. WHO. Vector-borne diseases. 2017. http://www.who.int/en/newsroom/fact-sheets/detail/vector-borne-diseases. Accessed 13 Mar 2018.

2. Gubler DJ. Human arbovirus infections worldwide. Ann NY Acad Sci. 2001;951:13-24.

3. Beckham JD, Tyler KL. Arbovirus infections. Continuum (Minneap, Minn). 2015;21(6 Neuroinfectious Disease):1599-611.

4. Laureti M, Narayanan D, Rodriguez-Andres J, Fazakerley JK, Kedzierski L. Flavivirus receptors: diversity, identity, and cell entry. Front Immunol. 2018;9:2180.

5. WHO. Dengue control strategies. 2017. http://www.who.int/dengu econtrol/control_strategies/en/. Accessed 30 Mar 2018.

6. Dash AP, Bhatia R, Sunyoto T, Mourya DT. Emerging and re-emerging arboviral diseases in Southeast Asia. J Vector Borne Dis. 2013;50:77-84

7. Weaver SC, Costa F, Garcia-Blanco MA, Ko Al, Ribeiro GS, Saade G, et al. Zika virus: history, emergence, biology, and prospects for control. Antiviral Res. 2016;130:69-80.

8. Romo H, Papa A, Kading R, Clark R, Delorey M, Brault AC. Comparative vector competence of North American Culex pipiens and Culex quinquefasciatus for African and European lineage 2 West Nile viruses. Am J Trop Med Hyg. 2018;98:1863-9.

9. Fragkoudis R, Chi Y, Siu RWC, Barry G, Attarzadeh-Yazdi G, Merits A, et al. Semliki Forest virus strongly reduces mosquito host defence signaling. Insect Mol Biol. 2008;17:647-56.

10. Huang Z, Kingsolver MB, Avadhanula V, Hardy RW. An antiviral role for antimicrobial peptides during the arthropod response to Alphavirus replication. J Virol. 2013;87:4272.

11. Lim XE, Lee SW, Madzokere TE, Herrero JL. Mosquitoes as suitable vectors for Alphaviruses. Viruses. 2018;10:84.

12. Sim C, Hong YS, Tsetsarkin KA, Vanlandingham DL, Higgs S, Collins FH. Anopheles gambiae heat shock protein cognate 70B impedes O'nyongnyong virus replication. BMC Genomics. 2007:8:231.

13. Franz AWE, Kantor AM, Passarelli AL, Clem RJ. Tissue barriers to arbovirus infection in mosquitoes. Viruses. 2015;7:3741-67.

14. Xi Z, Ramirez JL, Dimopoulos G. The Aedes aegypti toll pathway controls dengue virus infection. PLoS Pathog. 2008;4:e1000098.

15. Salazar MI, Richardson JH, Sánchez-Vargas I, Olson KE, Beaty BJ. Dengue virus type 2: replication and tropisms in orally infected Aedes aegypti mosquitoes. BMC Microbiol. 2007;7:9

16. Chauhan C, Behura SK, Debruyn B, Lovin DD, Harker BW, GomezMachorro $\mathrm{C}$, et al. Comparative expression profiles of midgut genes in dengue virus refractory and susceptible Aedes aegypti across critical period for virus infection. PLoS One. 2012;7:e47350.

17. Bennett KE, Flick D, Fleming KH, Jochim R, Beaty BJ, Black WC. Quantitative trait loci that control dengue-2 virus dissemination in the mosquito Aedes aegypti. Genetics. 2005;170:185-94.

18. Dostert C, Jouanguy E, Irving P, Troxler L, Galiana-Arnoux D, Hetru C, et al. The Jak-STAT signaling pathway is required but not sufficient for the antiviral response of Drosophila. Nat Immunol. 2005;6:946-53.

19. Hanson MA, Hamilton PT, Perlman SJ. Immune genes and divergent antimicrobial peptides in flies of the subgenus Drosophila. BMC Evol Biol. 2016;16:228

20. Satyavathi WV, Minz A, Nagaraju J. Nodulation: an unexplored cellular defense mechanism in insects. Cell Signal. 2014;26:1753-63.

21. Browne N, Heelan M, Kavanagh K. An analysis of the structural and functional similarities of insect hemocytes and mammalian phagocytes. Virulence. 2013;4:597-603

22. Souza-Neto JA, Sim S, Dimopoulos G. An evolutionary conserved function of the JAK-STAT pathway in anti-dengue defense. Proc Natl Acad Sci USA. 2009:106:17841-6.
23. Ramirez $\mathrm{JL}$, Dimopoulos $\mathrm{G}$. The Toll immune signaling pathway control conserved anti-dengue defenses across diverse Ae. aegypti strains and against multiple dengue virus serotypes. Dev Comp Immunol. 2010;34:625-9.

24. Sanders HR, Foy BD, Evans AM, Ross LS, Beaty BJ, Olson KE, et al. Sindbis virus induces transport processes and alters expression of innate immunity pathway genes in the midgut of the disease vector, Aedes aegypti. Insect Biochem Mol Biol. 2005;35:1293-307.

25. Angleró-Rodríguez YI, MacLeod HJ, Kang S, Carlson JS, Jupatanakul N, Dimopoulos G. Aedes aegypti molecular responses to Zika virus: modulation of infection by the Toll and Jak/Stat immune pathways and virus host factors. Front Microbiol. 2017;8:2050.

26. Tsuzuki S, Matsumoto H, Furihata S, Ryuda M, Tanaka H, Jae Sung E, et al. Switching between humoral and cellular immune responses in Drosophila is guided by the cytokine GBP. Nat Commun. 2014;5:4628.

27. Nainu F, Tanaka Y, Shiratsuchi A, Nakanishi Y. Protection of insects against viral infection by apoptosis-dependent phagocytosis. J Immunol. 2015;195:5696

28. Paradkar PN, Trinidad L, Voysey R, Duchemin J-B, Walker PJ. Secreted Vago restricts West Nile virus infection in Culex mosquito cells by activating the Jak-STAT pathway. Proc Natl Acad Sci USA. 2012;109:18915-20.

29. Lemaitre B, Hoffmann J. The host defense of Drosophila melanogaster. Annu Rev Immunol. 2007;25:697-743.

30. Goic B, Stapleford KA, Frangeul L, Doucet AJ, Gausson V, Blanc H, et al. Virus-derived DNA drives mosquito vector tolerance to arboviral infection. Nat Commun. 2016;7:12410.

31. Fire A, Xu S, Montgomery MK, Kostas SA, Driver SE, Mello CC. Potent and specific genetic interference by double-stranded RNA in Caenorhabditis elegans. Nature. 1998;391:806-11.

32. Ding S-W, Voinnet O. Antiviral immunity directed by small RNAs. Cell. 2007;130:413-26

33. Weber F, Wagner V, Rasmussen SB, Hartmann R, Paludan SR. Doublestranded RNA is produced by positive-strand RNA viruses and DNA viruses but not in detectable amounts by negative-strand RNA viruses. J Virol. 2006;80:5059-64.

34. Blair DC, Olson EK. The role of RNA interference (RNAi) in arbovirusvector interactions. Viruses. 2015;7:820-43.

35. Rand TA, Ginalski K, Grishin NV, Wang X. Biochemical identification of Argonaute 2 as the sole protein required for RNA-induced silencing complex activity. Proc Natl Acad Sci USA. 2004;101:14385-9.

36. Okamura K, Ishizuka A, Siomi H, Siomi MC. Distinct roles for Argonaute proteins in small RNA-directed RNA cleavage pathways. Genes Dev. 2004;18:1655-66.

37. Sánchez-Vargas I, Scott JC, Poole-Smith BK, Franz AWE, Barbosa-Solomieu V, Wilusz J, et al. Dengue virus type 2 infections of Aedes aegypti are modulated by the mosquito's RNA interference pathway. PLoS Pathog. 2009;5:e1000299.

38. Khoo CCH, Piper J, Sanchez-Vargas I, OIson KE, Franz AWE. The RNA interference pathway affects midgut infection- and escape barriers for Sindbis virus in Aedes aegypti. BMC Microbiol. 2010;10:130.

39. Hess AM, Prasad AN, Ptitsyn A, Ebel GD, Olson KE, Barbacioru C, et al. Small RNA profiling of dengue virus-mosquito interactions implicates the PIWI RNA pathway in anti-viral defense. BMC Microbiol. 2011;11:45.

40. Keene KM, Foy BD, Sanchez-Vargas I, Beaty BJ, Blair CD, Olson KE. RNA interference acts as a natural antiviral response to O'nyong-nyong virus (Alphavirus; Togaviridae) infection of Anopheles gambiae. Proc Natl Acad Sci USA. 2004;101:17240-5.

41. Tran T, Havlak P, Miller J. MicroRNA enrichment among short 'ultraconserved' sequences in insects. Nucleic Acids Res. 2006;34:e65.

42. Puthiyakunnon S, Yao Y, Li Y, Gu J, Peng H, Chen X. Functional characterization of three microRNAs of the Asian tiger mosquito, Aedes albopictus. Parasit Vectors. 2013;6:230

43. Lee YS, Nakahara K, Pham JW, Kim K, He Z, Sontheimer EJ, et al. Distinct roles for Drosophila Dicer-1 and Dicer-2 in the siRNA/miRNA silencing pathways. Cell. 2004:117:69-81.

44. Bavia L, Mosimann ALP, Aoki MN, Santos CN. A glance at subgenomic flavivirus RNAs and microRNAs in flavivirus infections. Virol J. 2016;13:84

45. Asgari S. Role of microRNAs in arbovirus/vector interactions. Viruses. 2014;6:3514-34. 
46. Lee M, Etebari K, Hall-Mendelin S, van den Hurk AF, Hobson-Peters J, Vatipally $S$, et al. Understanding the role of microRNAs in the interaction of Aedes aegypti mosquitoes with an insect-specific flavivirus. J Gen Virol. 2017;98:1892-903.

47. Saldaña MA, Etebari K, Hart CE, Widen SG, Wood TG, Thangamani $S$, et al. Zika virus alters the microRNA expression profile and elicits an RNAi response in Aedes aegypti mosquitoes. PLoS Negl Trop Dis. 2017; 11:e0005760.

48. Campbell CL, Harrison T, Hess AM, Ebel GD. MicroRNA levels are modulated in Aedes aegypti after exposure to Dengue-2. Insect Mol Biol. 2014;23:132-9.

49. Skalsky RL, Vanlandingham DL, Scholle F, Higgs S, Cullen BR. Identification of microRNAs expressed in two mosquito vectors, Aedes albopictus and Culex quinquefasciatus. BMC Genomics. 2010;11:119.

50. Carissimo G, Pondeville E, McFarlane M, Dietrich I, Mitri C, Bischoff E, et al. Antiviral immunity of Anopheles gambiae is highly compartmentalized, with distinct roles for RNA interference and gut microbiota. Proc Natl Acad Sci USA. 2015;112:E176.

51. Varjak M, Maringer K, Watson M, Sreenu VB, Fredericks AC, Pondeville $\mathrm{E}$, et al. Aedes aegypti: Piwi4 is a noncanonical PIWI protein involved in antiviral responses. mSphere. 2017;2:e00144.

52. Léger P, Lara E, Jagla B, Sismeiro O, Mansuroglu Z, Coppée JY, et al. Dicer-2- and Piwi-mediated RNA interference in Rift Valley fever virusinfected mosquito cells. J Virol. 2013;87:1631.

53. Miesen P, Girardi E, van Rij RP. Distinct sets of PIWI proteins produce arbovirus and transposon-derived piRNAs in Aedes aegypti mosquito cells. Nucleic Acids Res. 2015;43:6545-56.

54. Liu P, Dong Y, Gu J, Puthiyakunnon S, Wu Y, Chen X-G. Developmental piRNA profiles of the invasive vector mosquito Aedes albopictus. Parasit Vectors. 2016;9:524.

55. Siomi MC, Sato K, Pezic D, Aravin AA. PIWI-interacting small RNAs: the vanguard of genome defence. Nat Rev Mol Cell Biol. 2011;12:246.

56. Morazzani EM, Wiley MR, Murreddu MG, Adelman ZN, Myles KM. Production of virus-derived ping-pong-dependent piRNA-like small RNAs in the mosquito soma. PLoS Pathog. 2012;8:e1002470.

57. Schnettler E, Donald CL, Human S, Watson M, Siu RWC, McFarlane $M$, et al. Knockdown of piRNA pathway proteins results in enhanced Semliki Forest virus production in mosquito cells. J Gen Virol. 2013;94:1680-9.

58. Poirier EZ, Goic B, Tomé-Poderti L, Frangeul L, Boussier J, Gausson $\checkmark$, et al. Dicer-2-dependent generation of viral DNA from defective genomes of RNA viruses modulates antiviral immunity in insects. Cell Host Microbe. 2018;23(353-65):e8.

59. Nag DK, Kramer LD. Patchy DNA forms of the Zika virus RNA genome are generated following infection in mosquito cell cultures and in mosquitoes. J Gen Virol. 2017;98:2731-7.

60. Barletta ABF, Nascimento-Silva MCL, Talyuli OAC, Oliveira JHM, Pereira LOR, Oliveira PL, Sorgine MHF. Microbiota activates IMD pathway and limits Sindbis infection in Aedes aegypti. Parasit Vectors. 2017;10:103.

61. Zhang R, Zhu Y, Pang X, Xiao X, Zhang R, Cheng G. Regulation of antimicrobial peptides in Aedes aegypti Aag2 cells. Front Cell Infect Microbiol. 2017;7:22.

62. Hancock REW, Sahl H-G. Antimicrobial and host-defense peptides as new anti-infective therapeutic strategies. Nat Biotechnol. 2006;24:1551.

63. Yi H-Y, Chowdhury M, Huang Y-D, Yu X-Q. Insect antimicrobial peptides and their applications. Appl Microbiol Biotechnol. 2014;98:5807-22.

64. Vizioli J, Bulet P, Hoffmann JA, Kafatos FC, Müller H-M, Dimopoulos G. Gambicin: a novel immune responsive antimicrobial peptide from the malaria vector Anopheles gambiae. Proc Natl Acad Sci USA. 2001:98:12630.

65. Bartholomay LC, Fuchs JF, Cheng LL, Beck ET, Vizioli J, Lowenberger C, et al. Reassessing the role of defensin in the innate immune response of the mosquito, Aedes aegypti. Insect Mol Biol. 2004;13:125-32.

66. Luplertlop N, Surasombatpattana P, Patramool S, Dumas E, Wasinpiyamongkol L, Saune L, et al. Induction of a peptide with activity against a broad spectrum of pathogens in the Aedes aegypti salivary gland, following infection with dengue virus. PLoS Pathog. 2011;7:e1001252.

67. Vizioli J, Bulet P, Charlet M, Lowenberger C, Blass C, Muller HM, et al. Cloning and analysis of a cecropin gene from the malaria vector mosquito, Anopheles gambiae. Insect Mol Biol. 2000;9:75-84.
68. Jupatanakul N, Sim S, Angleró-Rodríguez Yl, Souza-Neto J, Das S, Poti KE, et al. Engineered Aedes aegypti JAK/STAT pathway-mediated immunity to dengue virus. PLoS Negl Trop Dis. 2017;11:e0005187.

69. Behura SK, Gomez-Machorro C, Harker BW, deBruyn B, Lovin DD, Hemme RR, et al. Global cross-talk of genes of the mosquito Aedes aegypti in response to dengue virus infection. PLoS Negl Trop Dis. 2011;5:e1385.

70. McFarlane M, Arias-Goeta C, Martin E, O'Hara Z, Lulla A, Mousson L, et al. Characterization of Aedes aegypti innate-immune pathways that limit chikungunya virus replication. PLoS Negl Trop Dis. 2014;8:e2994.

71. Fros JJ, Liu WJ, Prow NA, Geertsema C, Ligtenberg M, Vanlandingham $D L$, et al. Chikungunya virus nonstructural protein 2 inhibits type I/II interferon-stimulated JAK-STAT signaling. J Virol. 2010;84:10877.

72. Duneau DF, Kondolf HC, Im JH, Ortiz GA, Chow C, Fox MA, et al. The Toll pathway underlies host sexual dimorphism in resistance to both Gramnegative and Gram-positive bacteria in mated Drosophila. BMC Biol. 2017;15:124.

73. Shin SW, Bian G, Raikhel AS. A toll receptor and a cytokine, Toll5A and Spz1C, are involved in toll antifungal immune signaling in the mosquito Aedes aegypti. J Biol Chem. 2006;281:39388-95.

74. Kim M, Lee JH, Lee SY, Kim E, Chung J. Caspar, a suppressor of antibacterial immunity in Drosophila. Proc Natl Acad Sci USA. 2006;103:16358-63.

75. Sim S, Dimopoulos G. Dengue virus inhibits immune responses in Aedes aegypti cells. PLoS One. 2010;5:e10678.

76. Kopan R, llagan MXG. The canonical notch signaling pathway: unfolding the activation mechanism. Cell. 2009;137:216-33.

77. Serrato-Salas J, Hernández-Martínez S, Martínez-Barnetche J, Condé R, Alvarado-Delgado A, Zumaya-Estrada F, et al. De novo DNA synthesis in Aedes aegypti midgut cells as a complementary strategy to limit dengue viral replication. Front Microbiol. 2018;9:801.

78. Sun J, Deng W-M. Notch-dependent downregulation of the homeodomain gene cut is required for the mitotic cycle/endocycle switch and cell differentiation in Drosophila follicle cells. Development. 2005;132:4299

79. Eleftherianos I, Revenis C. Role and importance of phenoloxidase in insect hemostasis. J Innate Immun. 2011;3:28-33.

80. Parikh GR, Oliver JD, Bartholomay LC. A haemocyte tropism for an arbovirus. J Gen Virol. 2009:90:292-6.

81. Cheng G, Cox J, Wang P, Krishnan MN, Dai J, Qian F, et al. A C-type lectin collaborates with a CD45 phosphatase homolog to facilitate West Nile virus infection of mosquitoes. Cell. 2010;142:714-25.

82. Sigle LT, Hillyer JF. Eater and draper are involved in the periostial haemocyte immune response in the mosquito Anopheles gambiae. Insect Mol Biol. 2018;27:429-38.

83. Castillo JC, Robertson AE, Strand MR. Characterization of hemocytes from the mosquitoes Anopheles gambiae and Aedes aegypti. Insect Biochem Mol Biol. 2006;36:891-903.

84. Hillyer JF, Strand MR. Mosquito hemocyte-mediated immune responses. Curr Opin Insect Sci. 2014;3:14-21.

85. Nakatogawa SI, Oda Y, Kamiya M, Kamijima T, Aizawa T, Clark KD, et al. A novel peptide mediates aggregation and migration of hemocytes from an insect. Curr Biol. 2009;19:779-85.

86. Rodriguez-Andres J, Rani S, Varjak M, Chase-Topping ME, Beck MH, Ferguson MC, et al. Phenoloxidase activity acts as a mosquito innate immune response against infection with Semliki Forest virus. PLoS Pathog. 2012;8:e1002977.

87. Martins GF, Serrão JE, Ramalho-Ortigão JM, Pimenta PFP. A comparative study of fat body morphology in five mosquito species. Mem Inst Oswaldo Cruz. 2011;106:742-7.

88. Barletta AB, Alves LR, Silva MC, Sim S, Dimopoulos G, Liechocki S, et al. Emerging role of lipid droplets in Aedes aegypti immune response against bacteria and dengue virus. Sci Rep. 2016;6:19928.

89. Martín-Acebes MA, Blázquez A-B, de Oya N, Escribano-Romero E, Saiz J-C. West Nile virus replication requires fatty acid synthesis but is independent on phosphatidylinositol-4-phosphate lipids. PLoS One. 2011;6:e24970.

90. Merino-Ramos T, Vázquez-Calvo Á, Casas J, Sobrino F, Saiz J-C, MartínAcebes MA. Modification of the host cell lipid metabolism induced by hypolipidemic drugs targeting the acetyl coenzyme A carboxylase impairs West Nile virus replication. Antimicrob Agents Chemother. 2016:60:307. 
91. Heaton NS, Perera R, Berger KL, Khadka S, Lacount DJ, Kuhn RJ, et al. Dengue virus nonstructural protein 3 redistributes fatty acid synthase to sites of viral replication and increases cellular fatty acid synthesis. Proc Natl Acad Sci USA. 2010;107:17345-50.

92. Perera R, Riley C, Isaac G, Hopf-Jannasch AS, Moore RJ, Weitz KW, et al Dengue virus infection perturbs lipid homeostasis in infected mosquito cells. PLoS Pathog. 2012;8:e1002584.

93. Mizushima N, Levine B, Cuervo MA, Klionsky DJ. Autophagy fights disease through cellular self-digestion. Nature. 2008;451:1069.

94. Bryant B, Raikhel AS. Programmed autophagy in the fat body of Aedes aegypti is required to maintain egg maturation cycles. PLoS One. 2011;6:e25502.

95. Nakamoto M, Moy RH, Xu J, Bambina S, Yasunaga A, Shelly SS, et al. Virus recognition by Toll-7 activates antiviral autophagy in Drosophila. Immunity. 2012;36:658-67.

96. Moy RH, Gold B, Molleston JM, Schad V, Yanger K, Salzano M-V, et al. Antiviral autophagy restricts Rift Valley fever virus infection and is conserved from flies to mammals. Immunity. 2014;40:51-65.

97. Shelly S, Lukinova N, Bambina S, Berman A, Cherry S. Autophagy is an essential component of Drosophila immunity against vesicular stomatitis virus. Immunity. 2009;30:588-98.

98. Zhang J, Lan Y, Li MY, Lamers MM, Fusade-Boyer M, Klemm E, et al. Flaviviruses exploit the lipid droplet protein AUP1 to trigger lipophagy and drive virus production. Cell Host Microbe. 2018;23:e5.

99. Kambris Z, Cook PE, Phuc HK, Sinkins SP. Immune activation by lifeshortening Wolbachia and reduced filarial competence in mosquitoes. Science. 2009:326:134.

100. Hoffmann AA, Montgomery BL, Popovici J, Iturbe-Ormaetxe I, Johnson $\mathrm{PH}$, Muzzi F, et al. Successful establishment of Wolbachia in Aedes populations to suppress dengue transmission. Nature. 2011;476:454.

101. Dong Y, Simões ML, Marois E, Dimopoulos G. CRISPR/Cas9 -mediated gene knockout of Anopheles gambiae FREP1 suppresses malaria parasite infection. PLoS Pathog. 2018;14:e1006898.

102. Carvalho DO, McKemey AR, Garziera L, Lacroix R, Donnelly CA, Alphey $L$, et al. Suppression of a field population of Aedes aegypti in Brazil by sustained release of transgenic male mosquitoes. PLoS Negl Trop Dis. 2015;9:e0003864.

103. Hilgenboecker K, Hammerstein P, Schlattmann P, Telschow A, Werren JH. How many species are infected with Wolbachia? - a statistical analysis of current data. FEMS Microbiol Lett. 2008;281:215-20.

104. World Mosquito Program. Eliminate dengue. 2017. http://www.elim natedengue.com/program. Accessed 3 Apr 2018

105. Hoffmann AA, Iturbe-Ormaetxe I, Callahan AG, Phillips BL, Billington K, Axford JK, et al. Stability of the wMel Wolbachia infection following invasion into Aedes aegypti populations. PLoS Negl Trop Dis. 2014;8:e3115.
106. Frentiu FD, Zakir T, Walker T, Popovici J, Pyke AT, van den Hurk A, et al. Limited dengue virus replication in field-collected Aedes aegypti mosquitoes infected with Wolbachia. PLoS Negl Trop Dis. 2014;8:e2688.

107. Carrington LB, Tran BCN, Le NTH, Luong TTH, Nguyen TT, Nguyen PT, et al. Field- and clinically derived estimates of Wolbachia-mediated blocking of dengue virus transmission potential in Aedes aegypti mosquitoes. Proc Natl Acad Sci USA. 2018;115:361.

108. Asad S, Parry R, Asgari S. Upregulation of Aedes aegypti Vago 1 by Wolbachia and its effect on dengue virus replication. Insect Biochem Mol Biol. 2018;92:45-52

109. Pan X, Zhou G, Wu J, Bian G, Lu P, Raikhel AS, et al. Wolbachia induces reactive oxygen species (ROS)-dependent activation of the Toll pathway to control dengue virus in the mosquito Aedes aegypti. Proc Natl Acad Sci USA. 2012;109:E23-31.

110. Caragata EP, Rancès E, Hedges LM, Gofton AW, Johnson KN, O'Neill SL, et al. Dietary cholesterol modulates pathogen blocking by Wolbachia. PLoS Pathog. 2013;9:e1003459.

111. Geoghegan V, Stainton K, Rainey SM, Ant TH, Dowle AA, Larson T, et al. Perturbed cholesterol and vesicular trafficking associated with dengue blocking in Wolbachia-infected Aedes aegypti cells. Nat Commun. 2017;8:526.

112. Amuzu HE, Tsyganov K, Koh C, Herbert RI, Powell DR, McGraw EA. Wolbachia enhances insect-specific flavivirus infection in Aedes aegypti mosquitoes. Ecol Evol. 2018;8:5441-54.

113. Dodson BL, Hughes GL, Paul O, Matacchiero AC, Kramer LD, Rasgon JL. Wolbachia enhances West Nile virus (WNV) infection in the mosquito Culex tarsalis. PLoS Negl Trop Dis. 2014;8:e2965.

114. Hughes GL, Rivero A, Rasgon JL. Wolbachia can enhance Plasmodium infection in mosquitoes: implications for malaria control? PLoS Pathog. 2014;10:e1004182.

115. Kistler KE, Vosshall LB, Matthews BJ. Genome engineering with CRISPRCas9 in the mosquito Aedes aegypti. Cell Rep. 2015;11:51-60.

116. Gantz VM, Jasinskiene N, Tatarenkova O, Fazekas A, Macias VM, Bier E, et al. Highly efficient Cas9-mediated gene drive for population modification of the malaria vector mosquito Anopheles stephensi. Proc Natl Acad Sci USA. 2015;112:E6736.

117. Li M, Bui M, Yang T, Bowman CS, White BJ, Akbari OS. Germline Cas9 expression yields highly efficient genome engineering in a major worldwide disease vector, Aedes aegypti. Proc Natl Acad Sci USA. 2017;114:E10540-9.

118. Itokawa K, Komagata O, Kasai S, Ogawa K, Tomita T. Testing the causality between CYP9M10 and pyrethroid resistance using the TALEN and CRISPR/Cas9 technologies. Sci Rep. 2016;6:24652.

119. Grigoraki L, Puggioli A, Mavridis K, Douris V, Montanari M, Bellini R, et al. Striking diflubenzuron resistance in Culex pipiens, the prime vector of West Nile Virus. Sci Rep. 2017;7:11699.
Ready to submit your research? Choose BMC and benefit from:

- fast, convenient online submission

- thorough peer review by experienced researchers in your field

- rapid publication on acceptance

- support for research data, including large and complex data types

- gold Open Access which fosters wider collaboration and increased citations

- maximum visibility for your research: over 100M website views per year

At $\mathrm{BMC}$, research is always in progress.

Learn more biomedcentral.com/submissions 